\title{
Fenomenología
}

\section{Más allá de las “indiciones”. Reflexión sobre la comunicación a partir de una vivencia}

\section{Beyond the injections. A reflection based on a experience about the communicaction}

\section{Além das injecções. Reflexão sobre a comunicação de uma experiência}

Raquel A. Díaz Alonsoํㄹ David Palacios Martínez²

${ }^{1}$ Diplomada Universitaria en Enfermería. Servicio de Medicina Interna.

Hospital Universitario de Fuenlabrada. Fuenlabrada, Madrid, España.

${ }^{2}$ Médico de Familia. Servicio Madrileño de Salud - SERMAS. Dirección Asistencial Sur de Madrid.

C.S. San Blas (Parla, Madrid, España).

Cómo citar este artículo en edición digital: Díaz Alonso, R.A. y Palacios Martínez, D. (2014) Más allá de las "indiciones".

Reflexión sobre la comunicación a partir de una vivencia. Cultura de los Cuidados (Edición digital) 18, 40. Disponible en: http://dx.doi.org/10.7184/cuid.2014.40.02>

Correspondencia: Raquel Asunción Díaz Alonso. C/ Nazaret, $N^{\circ} 6$, Portal 2, 2º B. Parla C.P. 28980. Madrid. E-mail: raquelda_parla@hotmail.com

Recibido: 20/07/2014; Aceptado: 14/09/2014

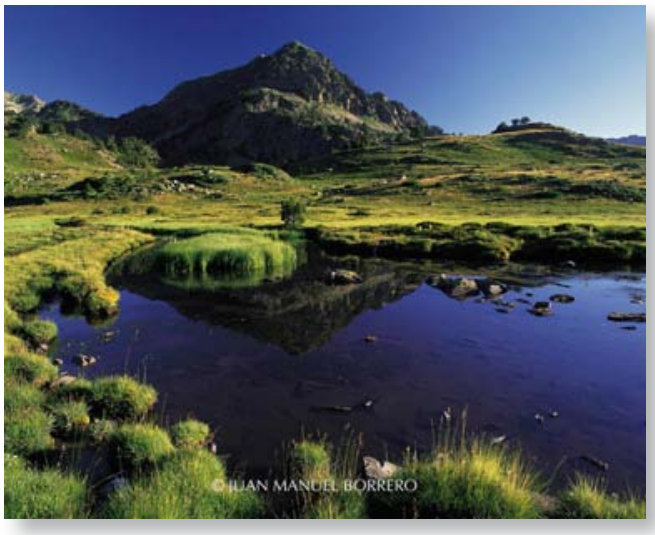

\section{ABSTRACT}

The aim of this paper is to describe a reflection based on an experience about the communication. In this experience, the interpretation of the message was essential. The first step of the sanitary intervention is the interview with the patient. After that, we can detect their needs and begin to act properly. The inadequate interpretation of the data obtained by the interview may conduct to un- expected outcomes. Frequently, the patients reinterpret or even reinvent the names of the drugs which are part of their usual treatment. Sometimes, it may also happen with the name of their diseases or the description of their underlying pathology. They may be influenced by several factors (cultural, geographical, etc). Decipher its true meaning may pose a real challenge sometimes. A recent conversation with my grandmother led me to reflect on the relationship between healthcare professionals and patients. This reflection is presented in this paper.

Key words: holistic nursing; nurse-patients relations; patient medication knowledge; ethics, nursing; philosophy, nursing.

\section{RESUMO}

O objetivo deste artigo é descrever uma reflexão originou-se de uma vivência. Nele, a interpretação da mensagem era indispensável. 
O primeiro passo na área da saúde é a entrevista com o paciente. Depois disso, podemos identificar suas necessidades e comece a agir corretamente. A interpretação inadequada dos dados obtidos através da entrevista pode levar a diferentes dos resultados esperados. Muitas vezes, os pacientes reinterpretam ou mesmo reinventar os nomes das drogas, doenças, etc. Às vezes isso pode acontecer também com a descrição da patologia subjacente. Esses fatos tendem a ser influenciado por vários fatores (culturais, geográficas, etc.). Decifrar o seu verdadeiro significado pode representar um desafio às vezes. Uma conversa recente com a minha avó me levou a refletir sobre a relação profissional entre apresentado neste trabalho.

Palavras-chave: enfermagem holística, relações enfermeiro-paciente, conhecimento do paciente sobre a medicação, ética em enfermagen, filosofía em enfermagem.

\section{RESUMEN}

El propósito de este trabajo es describir una reflexión originada a partir de una vivencia. En ella, la interpretación del mensaje fue indispensable. El primer paso de la asistencia sanitaria es la entrevista con el paciente. Tras ello, podemos detectar sus necesidades y empezar a actuar adecuadamente. La interpretación inadecuada de los datos obtenidos mediante la entrevista puede conducir a resultados diferentes de los esperados. Con cierta frecuencia, los pacientes reinterpretan o incluso reinventan los nombres de los fármacos, de las enfermedades, etc. En ocasiones puede suceder también con la descripción de la patología subyacente. Estos hechos suelen estar influenciados por diversos factores (culturales, geográficos, etc.). Descifrar su verdadero significado puede llegar a suponer un verdadero reto en algunas ocasiones. Una conversación reciente con mi abuela me condujo a la reflexión sobre la relación profesional sanitariopaciente presentada en este trabajo.

Palabras clave: enfermería holística, relaciones enfermero-paciente, conocimiento de la medicación por el paciente, ética en enfermería, filosofía en enfermería.

Mi abuela, natural de un pequeño pueblo de Toledo (España) y costurera durante décadas, me llamó hace unos días. Quería que la acompañara “al trin-trón”. Esta expresión simpática se refiere al Sintrom ${ }^{\circledR}$ (marca comercial del acenocumarol en España). Su expresión me hizo sonreír. En realidad, ella quería que la acompañase al Centro de salud para realizarse el control de INR. Este hecho me provocó algunas reflexiones que desearía compartir.

Como profesionales sanitarios, una de nuestras atribuciones en el trabajo diario es la interpretación. La mayoría de las veces, nuestro cerebro procesa inmediatamente la traducción de lo que la persona nos quiere decir. En otras ocasiones, tenemos que esforzarnos. Incluso iniciar una investigación con el paciente para descubrir a qué se refiere. No son pocas las situaciones en las que no podemos evitar sonreír, por lo divertidas que nos resultan la palabra o la expresión utilizada.

Ejemplos hay miles, muchos de ellos derivados de la deformación popular de nombres o expresiones complejos. "Menos dos" (Ventimask). "Quince" (esguince). "Chinchón” o "trin-trón” (Sintrom $\left.{ }^{\circledast}\right)$. "Indición" (inyección). "Atontolol" (atenolol). "Anapril" (enalapril). "Vengo por los cuchillos de la garganta" (odinofagia/faringitis). "Tengo cita con el Doctor Rino" (otorrinolaringólogo). "Estoy con las angustias" (en función de la zona geográfica de procedencia del paciente, "angustias" cor- 
responde a ansiedad, náuseas y/o vómitos, disnea o dolor abdominal). "Los perros/lobos agarrados" (dolor). "Vengo con el tontillo" (no se refería al acompañante, sino al temblor en las manos).

A veces nos sorprenden las reflexiones humanas, muchas de ellas también procedentes del arraigo popular. “Cómo va a saber la pastilla dónde tiene que ir, con lo pequeña que es?". "Yo no soy diabética. El problema es que me da alergia el azúcar". "El niño sigue con diarrea a pesar de la dieta blanda. Sólo le damos natillas". "Vengo porque desde ayer tengo las piernas azules", porque le habían desteñido sus vaqueros nuevos.

La salud, como otros tantos ámbitos de la vida humana, es un campo del que todos tenemos alguna noción. Ya sea por curiosidad, por necesidad, por haber vivido de cerca una enfermedad. Lo mismo pasa, por ejemplo, con la mecánica de vehículos, con la agricultura, con la confección de ropa. Por eso, analizando nuestro comportamiento desde esta perspectiva, entiendo la sonrisa del mecánico cuando le pedí que me rellenara el "líquido azul de los cristales del coche". O la del frutero cuando compré dos kilos de brócoli en noviembre (amablemente me explicó que es la época en la que más caro está). O la de mi abuela cuando traté de entrar en un vestido tres tallas menores de lo necesario alegando que "en el dobladillo tendrá tela suficiente".

No podemos ser expertos todos en todo. A veces tenemos que esforzarnos en interpretar lo que quieren transmitirnos otras personas. Del mismo modo que, en otras ocasiones, alguien tiene que esforzarse para interpretar lo que nosotros queremos transmitirle. En ocasiones puede resultarnos muy difícil lograr comunicarnos de forma efectiva, pero es muy gratificante cuando lo logramos.

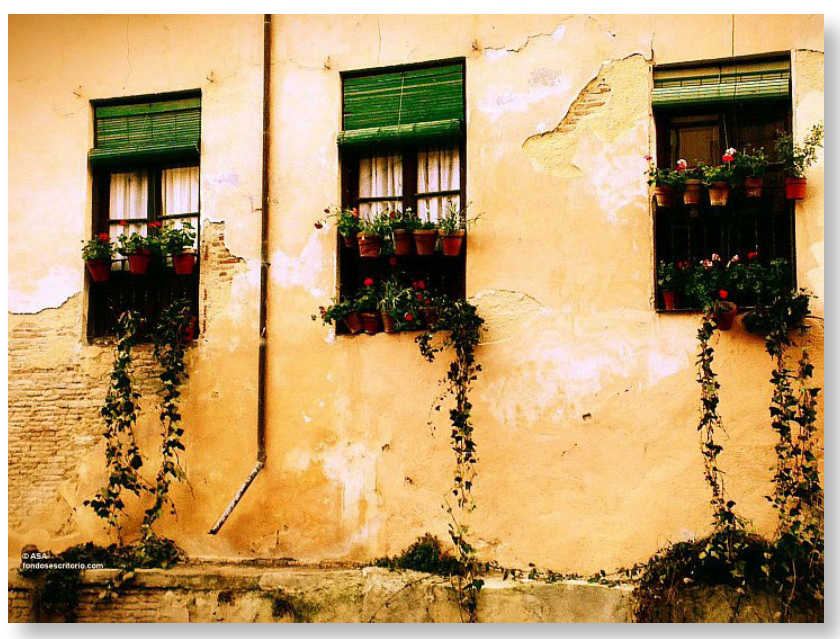

